\title{
Correction to: Adverse drug reaction causality assessment tools for drug-induced Stevens-Johnson syndrome and toxic epidermal necrolysis: room for improvement
}

\author{
Jennifer L. Goldman ${ }^{1} \cdot$ Wen-Hung Chung ${ }^{2}$ - Brian R. Lee ${ }^{3} \cdot$ Chun-Bing Chen ${ }^{2} \cdot$ Chun-Wei Lu ${ }^{2}$. \\ Wolfram Hoetzenecker ${ }^{4}$. Robert Micheletti ${ }^{5}$. Sally Usdin Yasuda ${ }^{6}$. David J. Margolis ${ }^{5,7} \cdot$ Neil H. Shear ${ }^{8}$. \\ Jeffery P. Struewing ${ }^{9} \cdot$ Munir Pirmohamed $^{10}$
}

Published online: 29 June 2019

(C) Springer-Verlag GmbH Germany, part of Springer Nature 2019

\section{Correction to: European Journal of Clinical Pharmacology} https://doi.org/10.1007/s00228-019-02670-9

The correct name of the 9th Author is David J. Margolis.

The original article was corrected.

Publisher's note Springer Nature remains neutral with regard to jurisdictional claims in published maps and institutional affiliations.

The online version of the original article can be found at https://oi.org/ 10.1007/s00228-019-02670-9

Jennifer L. Goldman

jlgoldman@cmh.edu

1 Department of Pediatrics, Divisions of Pediatric Infectious Diseases \& Clinical Pharmacology, Children's Mercy Hospitals \& Clinic, 2401 Gillham Rd., Kansas City, MO 64108, USA

2 Department of Dermatology, Drug Hypersensitivity Clinical and Research Center, Chang Gung Memorial Hospital, Keelung and Linkou Branches, College of Medicine, Chang Gung University, Taipei, Taiwan

3 Division of Health Services and Outcomes Research, Children's Mercy Hospitals \& Clinics, Kansas City, USA

4 Department of Dermatology, University Hospital Linz, Linz, Austria
5 Department of Dermatology, University of Pennsylvania, Philadelphia, USA

6 Food and Drug Administration, Silver Spring, USA

7 Department of Biostatistics, Epidemiology and Informatics, University of Pennsylvania, Philadelphia, USA Division of Dermatology, Department of Medicine, Sunnybrook Health Sciences Center and University of Toronto, Toronto, Canada

9 Division of Genomic Medicine, National Human Genome Research Institute, National Institutes of Health, Bethesda, USA

10 Department ofMolecular and Clinical Pharmacology, University of Liverpool, Liverpool, UK 